\title{
Contaminación por metales pesados en los sedimentos acumulados sobre el corredor vial Bogotá - Soacha
}

\section{Heavy metal pollution in the sediments accumulated on Bogotá - Soacha highway (Colombia)}

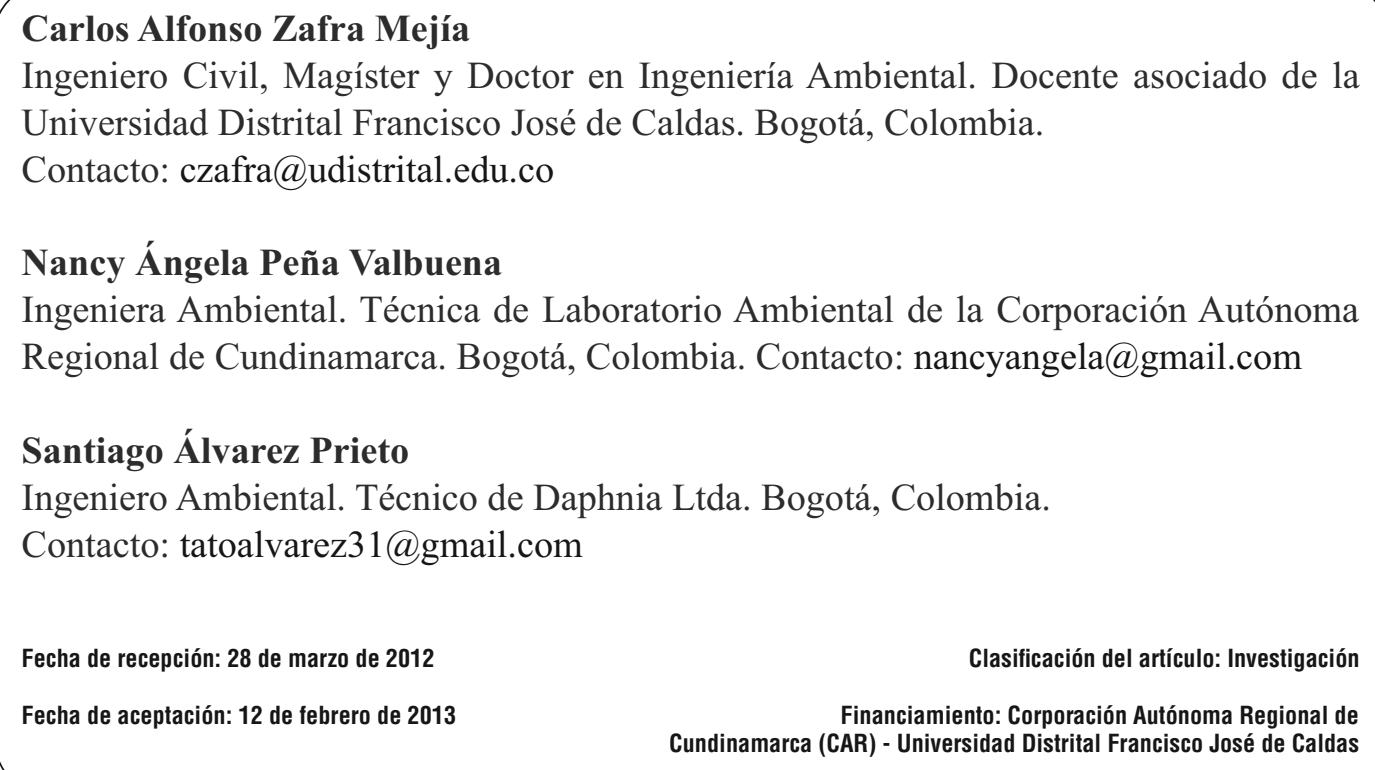

Palabras clave: contaminación, calidad del agua, escorrentía.

Key words: pollution, water quality, runoff.

\section{RESUMEN}

Los sedimentos viales acumulados en tiempo seco llevan consigo elementos metálicos que afectan los recursos hídricos cuando son transportados por la escorrentía, y deterioran la calidad del aire cuando son suspendidos por el viento y la turbulencia inducida por el tráfico. Este artículo presenta los resultados de la evaluación de la contaminación por metales pesados asociados con el sedimento vial de dos zonas (i.e., Zonas 1 y 2) del corredor Bogotá - Soacha en Colombia. El sedimento vial en cada zona de investigación se recolectó por barrido directo sobre una superficie de área de $0,25 \mathrm{~m}^{2} \mathrm{y}$ durante un periodo de cuatro meses. Se determinaron las concentraciones de $\mathrm{Mn}, \mathrm{Fe}, \mathrm{Cu}, \mathrm{Zn}, \mathrm{As}, \mathrm{Cd}$, Ba y $\mathrm{Pb}$ para la fracción de tamaño menor de $250 \mu \mathrm{m}$ del sedimento vial por medio de espectrometría de absorción atómica de llama y de plasma inductivamente acoplado con 


\section{investigación |}

espectrometría de masas. Los resultados sugirieron que el origen principal de los metales pesados fueron las fuentes móviles (por ejemplo, por desgaste de llantas, pastillas de frenos y autopartes). Adicionalmente, mostraron que las concentraciones de $\mathrm{Pb}$ y Zn fueron elevadas a partir de la legislación utilizada como referencia; con respecto de los suelos urbanos, los metales pesados que sobrepasaron los límites permisibles fueron los siguientes: $\mathrm{Pb}(21,7 \%$ y 87,0 $\%$ de las veces en las Zonas 1 y 2 , respectivamente) y Zn $(84,8 \%$ y $82,6 \%$ de las veces en las Zonas 1 y 2 , respectivamente).

\section{ABSTRACT}

Road sediments accumulated in dry weather carry metallic elements, which affects water resources when transported by runoff and deteriorates the air quality when suspended by wind and the turbulence induced by traffic. This paper presents the results of a pollution assessment on heavy metal particles associated to road sediment from two particular areas (i.e. Zones 1 and 2) of the Bogotá-Soacha highway (Colombia). Road sediment was collected in each area by direct sweep over a surface of $0.25 \mathrm{~m}^{2}$ and for a period of four months. The concentration of Mn, $\mathrm{Fe}, \mathrm{Cu}, \mathrm{Zn}, \mathrm{As}, \mathrm{Cd}, \mathrm{Ba}$ and $\mathrm{Pb}$ was determined per size fraction (less than $250 \mu \mathrm{m}$ from road sediment). These figures were obtained by means of flame atomic absorption spectrometry and also by using inductively coupled plasma mass spectrometry. The results suggest that the main origin of heavy metals were the mobile sources (i.e. the wear of tires, brake pads and automotive parts). In addition, it is shown that the concentration of $\mathrm{Pb}$ and $\mathrm{Zn}$ was higher than that allowed by current regulations (used as reference). With regard to urban soils, heavy metals that exceeded the permissible limits were as follows: $\mathrm{Pb}$ $(21.7 \%$ and $87.0 \%$ of the time in Zones 1 and 2, respectively) and $\mathrm{Zn}(84.8 \%$ and $82.6 \%$ of the time in Zones 1 and 2, respectively).

\section{INTRODUCCIÓN}

La presencia de elementos metálicos en zonas remotas ha despertado el interés por estudiar su transporte en el aire, agua y suelo [1], [2]. En estudios realizados a nivel mundial se han encontrado elevadas concentraciones de metales pesados en el agua, el suelo, la biota y los sedimentos lacustres [3]. En estas áreas la principal vía de ingreso de los metales ha sido la atmósfera. Esto se debió a que algunos metales tuvieron una elevada volatilidad que facilitó su transporte, como fue el caso de $\mathrm{Hg}$ y $\mathrm{Pb}$. No obstante, los metales que no fueron volátiles (por ejemplo, $\mathrm{Cu}$ y $\mathrm{Zn}$ ) se encontraban asociados con partículas finas en las masas de aire y, por tanto, estuvieron sujetos al transporte atmosférico [4].

Actualmente se tiene evidencia de la contaminación atmosférica generada por el transporte y la industria, la cual ha llegado a niveles críticos en los

principales corredores viales de Colombia [5]. Los problemas de contaminación que se presentan en Bogotá D.C. y el municipio de Soacha (Colombia) se deben principalmente al elevado tráfico vehicular, así como también a la presencia de industrias y actividades mineras. En este sentido, en algunas investigaciones [2], [6] se ha demostrado que la actividad industrial y el tráfico cumplen un rol importante en la formación de partículas, y participan directa e indirectamente en la formación de aerosoles secundarios.

Las superficies viarias representan solo una pequeña parte del paisaje urbano, no obstante el aumento de su área superficial impermeable contribuye con significativas cargas de contaminantes durante los eventos de lluvia [7]. Los sedimentos viarios acumulados en tiempo seco llevan consigo elementos metálicos que afectan los recursos hídricos cuando son transportados por la escorrentía [8], [9], y pue- 
den afectar la calidad del aire del entorno cuando son suspendidos por el viento y la turbulencia inducida por el tráfico [10], [11].

La contaminación causada por el agua de escorrentía se constituye en un problema sobre las áreas urbanas. Algunas investigaciones [12] - [14] han demostrado que este tipo de aguas contienen elevadas concentraciones de contaminantes, de manera semejante a las aguas residuales urbanas. Por otra parte, la escorrentía superficial urbana es considerada como una fuente de contaminación de difícil localización (difusa) debido a que su vertido es intermitente y ligado a la lluvia, la cual es un fenómeno aleatorio, variable en el tiempo y difícil de muestrear [15]. De esta manera, la contaminación superficial por metales pesados requiere de especial atención debido a sus efectos tóxicos sobre la salud del hombre, los recursos hídricos, los suelos y la atmósfera [16].

En Colombia hasta el momento no se han realizado estudios que evalúen la contaminación por metales pesados asociados con el sedimento viario, a pesar de que existen importantes corredores viales (por ejemplo, Bogotá, D.C. - Soacha). En este sentido, es preciso desarrollar estudios que evalúen el comportamiento de los metales pesados asociados con el sedimento viario y los efectos sobre la salud a partir de las concentraciones metálicas detectadas. De esta manera se podrá tener un punto de partida para el establecimiento de normas de regulación para este tipo de contaminantes, como también para el diseño e implementación de técnicas de minimización y control por parte de los organismos de control ambiental.

El objetivo principal de la investigación fue evaluar el contenido de los metales pesados asociados con el sedimento del corredor vial Bogotá - Soacha. Los investigadores pretendieron sugerir el origen de los metales pesados y evaluar la relación entre los condicionantes climatológicos y el contenido metálico asociado con el sedimento viario. Este artículo inicia con una descripción de los materiales y métodos utilizados para la caracterización metálica del

\section{|investigación}

sedimento viario. Seguidamente, se presentan las concentraciones de los metales pesados asociados con el sedimento viario y una comparación con los límites establecidos por la legislación tomada como referencia. Finalmente, se exponen las principales conclusiones de la investigación.

\section{METOdOLOGÍA}

\subsection{Descripción del lugar de investigación}

El lugar de investigación se localizó en el corredor vial Bogotá - Soacha, perteneciente al área metropolitana de la ciudad de Bogotá, D.C. (Colombia), en el municipio de Soacha (figura 1). La temperatura promedio anual fue de $14{ }^{\circ} \mathrm{C}$ y la precipitación media anual registrada fue de $777 \mathrm{~mm}$. El área de estudio tiene una elevación media de 2600 m.s.n.m. Las superficies viarias en evaluación tuvieron un elevado tráfico con predominancia de vehículos de carga y de transporte público. Adicionalmente, presentaron una elevada actividad industrial y contaron con gran cantidad de edificaciones a los costados de las vías.

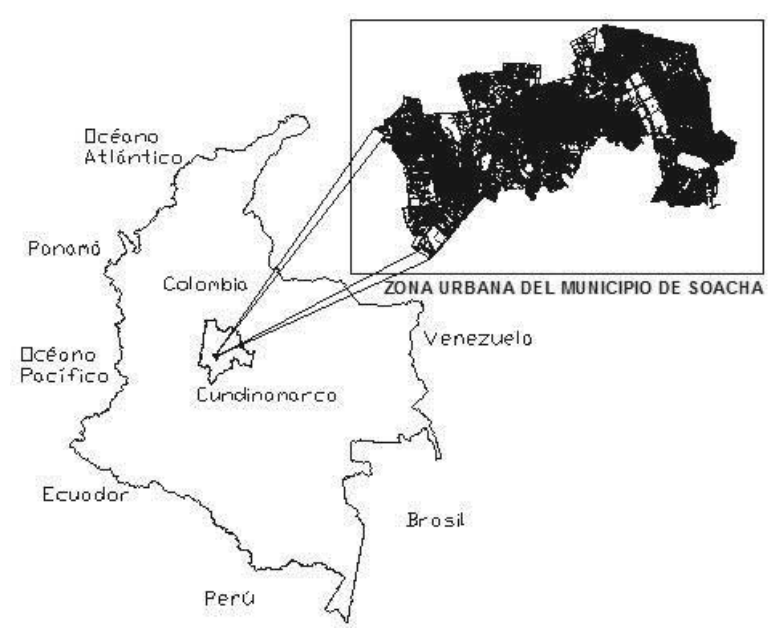

Figura 1. Localización del área de investigación.

Fuente: elaboración propia.

Dentro del área de estudio se seleccionaron dos zonas de muestreo (figura 2 y figura 3). La Zona 1, localiza- 


\section{investigación}

da en el centro urbano del municipio de Soacha en las vías adyacentes al hospital Mario Gaitán Yanguas y a una distancia de $600 \mathrm{~m}$ con respecto de la Autopista Sur Bogotá - Soacha. En esta zona el uso del suelo en orden de importancia fue residencial, comercial e institucional. La superficie viaria no contó con líneas de aparcamiento, sin embargo durante el periodo de muestreo se observaron vehículos estacionados a un costado (figura 2). El sistema de drenaje estuvo constituido por sumideros localizados en las bocas-calles, es decir, no existieron cunetas para el transporte de la escorrentía viaria. Las principales características de la Zona 1 se presentan en la tabla 1.

La Zona 2 correspondió al corredor vial Autopista Sur Bogotá - Soacha, frente a las bodegas de la empresa Almacafé en la zona industrial Santa Ana del municipio de Soacha (figura 2). Esta zona tiene un uso del suelo de tipo industrial, el sistema de drenaje de la vía está constituido por una serie de sumideros localizados cada $55 \mathrm{~m}$, los cuales conducen las aguas de escorrentía hacia unos canales que desembocan en el río Bogotá. La superficie viaria no posee cunetas para el transporte de la escorrentía. Adicionalmente, la zona de investigación se caracteriza por poseer áreas abiertas (poco edificada). Las principales características de la Zona 2 se presentan en la tabla 1.

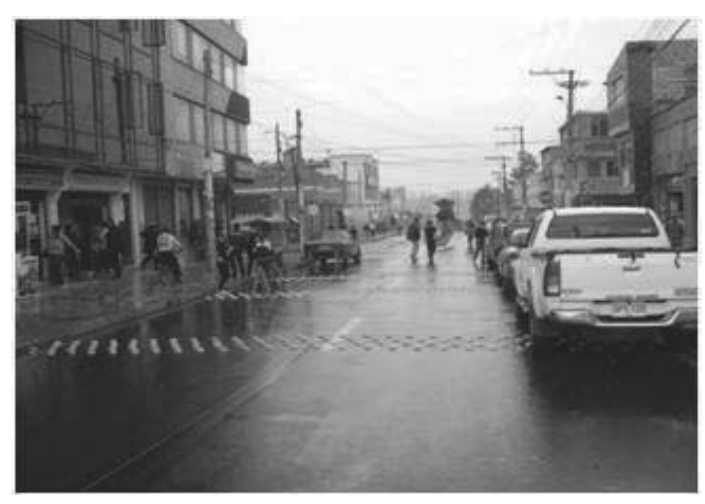

Figura 2. Fotografías de las superficies viarias de muestreo Zona 1 Fuente: elaboración propia.

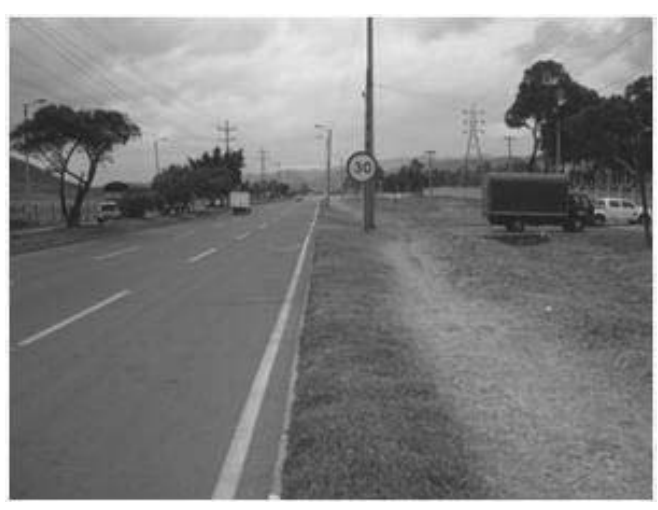

Figura 3. Fotografías de las superficies viarias de muestreo Zona 2. Fuente: elaboración propia.

Tabla 1. Características de las zonas de muestreo.

\begin{tabular}{|c|c|c|}
\hline Característica & Zona 1 & Zona 2 \\
\hline $\begin{array}{c}\text { Densidad } \\
\text { residencial } \\
\text { (habitantes/ha) }\end{array}$ & Alta (600) & $\begin{array}{l}\text { Baja } \\
(150)\end{array}$ \\
\hline $\begin{array}{c}\text { Líneas de tráfico/ } \\
\text { parqueo }\end{array}$ & 1a/ninguna & $\begin{array}{c}2^{\mathrm{b}} / \\
\text { ninguna }\end{array}$ \\
\hline Longitud viaria $(\mathrm{m})$ & 120 & 500 \\
\hline $\begin{array}{c}\text { Pendiente } \\
\text { longitudinal/ } \\
\text { transversal (\%) }\end{array}$ & $2 / 4$ & $1 / 4$ \\
\hline $\begin{array}{l}\text { Tipo/textura del } \\
\text { pavimento }\end{array}$ & Asfalto/rugoso & $\begin{array}{c}\text { Asfalto/ } \\
\text { rugoso }\end{array}$ \\
\hline $\begin{array}{c}\text { Tráfico } \\
\text { promedio diario } \\
\text { (Vehículos/d) }\end{array}$ & 2750 & 40100 \\
\hline $\begin{array}{c}\text { Tráfico máximo } \\
\text { horario } \\
\text { (Vehículos/h) }\end{array}$ & 570 & 6700 \\
\hline $\begin{array}{c}\text { Velocidad } \\
\text { promedio }(\mathrm{km} / \mathrm{h})\end{array}$ & $10-30$ & $60-80$ \\
\hline $\begin{array}{l}\text { Composición del } \\
\text { tráfico } \\
\text { Zona } 1 / \text { Zona } 2 \\
(\%)\end{array}$ & $\begin{array}{c}\text { carros: } 65 / 62 \text {; camiones } \\
\text { ligeros: } 0 / 7,25 \text {; camiones } \\
\text { sin remolque: } 0 / 5,40 ; \\
\text { camiones con remolque: } \\
\text { 0/7,35; buses: } 35 / 18\end{array}$ & \\
\hline
\end{tabular}

Nota: ${ }^{a}$ : uso ocasional como línea de aparcamiento; ${ }^{\text {b: }}$ tres carriles por cada sentido del tráfico.

Fuente: elaboración propia. 
Los datos climatológicos de precipitación, temperatura, velocidad y dirección del viento se tomaron de las estaciones de monitoreo Kennedy, Carvajal-Sony y Tunal, administradas por la Secretaría Distrital de Ambiente de Bogotá (SDA) y localizadas a una distancia promedio de $6450 \mathrm{~m}$ con respecto de las superficies viarias en evaluación. Adicionalmente, se utilizaron los datos de precipitación de las estaciones de la Corporación Autónoma Regional de Cundinamarca (CAR): Almacafé y Fute, localizadas a una distancia promedio de $1750 \mathrm{~m}$ del área de investigación.

\subsection{Sistema de muestreo}

La recolección del sedimento viario se realizó durante un periodo de cuatro meses (14/01/2010 - 14/05/2010), en horas de la mañana (entre las 9:00 - 11:00 a. m.) y con una intensidad semanal de tres veces (por ejemplo, para un total de 96 muestras). El sedimento se recolectó a un costado de las superficies viarias (distancia al bordillo: 0,40 $\mathrm{m})$, teniendo en cuenta que la superficie estuviera seca y evitando la recolección sobre la pintura de demarcación de la vía (figura 4). El tamaño de la superficie de muestreo se controló con un marco de madera de $0,50 \times 0,50 \mathrm{~m}\left(0,25 \mathrm{~m}^{2}\right)$. El sedimento recolectado se almacenó en bolsas herméticas, y los sitios de recolección fueron debidamente identificados para no repetir los puntos de muestreo.

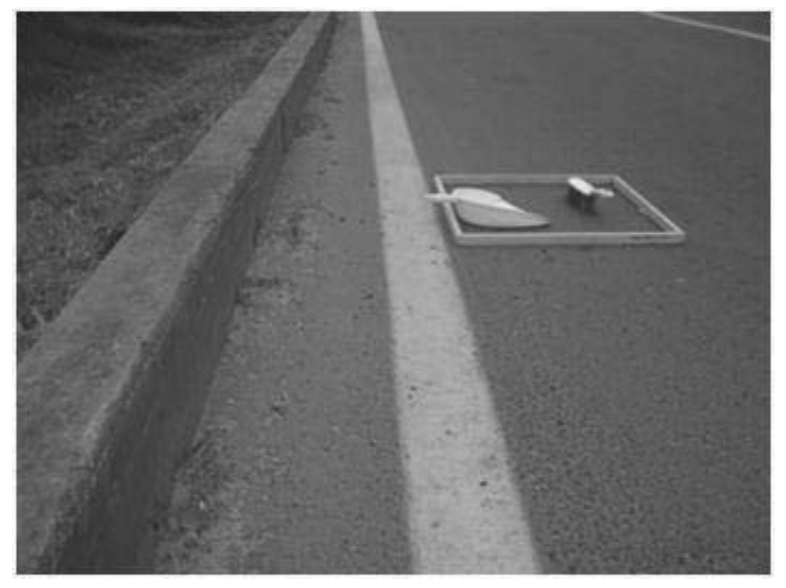

Figura 4. Sistema de muestreo para el sedimento viario Fuente: elaboración propia.

\subsection{Análisis de laboratorio}

Se realizó un pre-tratamiento de las muestras, el cual consistió en la determinación del contenido de humedad del sedimento viario (ASTM D2216) [17]. Seguidamente, se realizó un tamizado para separar la fracción de tamaño menor a $250 \mu \mathrm{m}$ y someterla a una extracción ácida en agua regia (ISO 11466) [18]. A partir del extracto obtenido, se determinó el contenido de Fe utilizando un equipo (UNICAM modelo 989) de espectrometría de absorción atómica de llama (ISO 11047) [19], y se utilizó un equipo de plasma inductivamente acoplado con espectrometría de masas (ICP-MS VARIAN 820) para la determinación de $\mathrm{Pb}, \mathrm{Zn}, \mathrm{Cu}, \mathrm{Ba}, \mathrm{As}, \mathrm{Mn}$ y Cd (ISO 11047) [19].

\section{RESULTADOS}

\subsection{Caracterización climatológica}

La precipitación durante el periodo de muestreo correspondió a un $31,7 \%$ de la precipitación total registrada para el mismo año $(777 \mathrm{~mm})$. Se identificaron dos épocas a partir de la variación en la precipitación: un periodo de tiempo seco entre los meses de enero y febrero, y un periodo de lluvias entre los meses de abril y mayo. En el área de investigación la velocidad media del viento durante el periodo de muestreo fue de $1,19 \mathrm{~m} / \mathrm{s}$ (variación entre: $0,71-2,2 \mathrm{~m} / \mathrm{s}$ ). Con respecto a la dirección predominante del viento, se observó que este provino del suroccidente. A partir de lo anterior, se sugirió que la dispersión de los contaminantes metálicos presentes sobre las superficies viarias de investigación probablemente se dio hacia el nororiente; es decir, desde el municipio de Soacha hacia la ciudad de Bogotá.

\subsection{Carga de sedimento viario}

La humedad del sedimento viario en la Zona 2 tendió a ser mayor durante el periodo de muestreo $(3,1 \%)$, con respecto de la Zona $1(2,6 \%)$. Los resultados sugirieron que la diferencia existente en la humedad asociada con el sedimento probablemente 


\section{investigación |}

se debió a la menor pendiente viaria de la Zona 2 $(1,0 \%)$. Es decir, a medida que disminuye la pendiente de la vía aumenta el tiempo de residencia del agua de escorrentía sobre esta.

La carga de sedimento viario en las Zonas 1 y 2 fue de 92,5 y $80,1 \mathrm{~g} / \mathrm{m}^{2}$, respectivamente. Como se pudo observar, la Zona 1 presentó la mayor cantidad de sedimento por unidad de superficie. La diferencia existente en la densidad residencial y la mayor exposición a la acción del viento de la Zona 2, sugirieron la inferior carga total depositada sobre la misma (tabla 1). Ball et ál. [7] encontraron que velocidades del viento superiores a $5,8 \mathrm{~m} / \mathrm{s}$ producían una suspensión de las partículas acumuladas sobre la calzada, y Barkdoll et ál. [20] mostraron que una partícula de $246 \mu \mathrm{m}$ podía ser suspendida por masas de aire con velocidades superiores a 2,2 m/s.

\subsection{Concentración de metales pesados en el sedimento viario}

Los resultados revelaron que los metales pesados más abundantes en las zonas de estudio fueron en orden de magnitud Fe, $\mathrm{Ba}, \mathrm{Pb}, \mathrm{Mn}$ y $\mathrm{Zn}$ (tabla 2). Estos resultados fueron similares a los encontrados por Zafra et ál. [15] y Machado et ál. [21] en el sedimento viario de las ciudades de Torrelavega (España) y Maracaibo (Venezuela), respectivamente.

Al comparar las dos zonas de estudio en la ciudad de Soacha, la Zona 2 tendió a presentar las mayores concentraciones de elementos metálicos. En promedio, el 87,5 \% de las concentraciones de la Zona 2 fueron mayores que las determinadas en la Zona 1 con respecto al valor medio de concentración para cada fracción de tamaño (tabla 2). Las concentraciones metálicas de la Zona 2 fueron en promedio 2,26 veces mayores que las concentraciones de la Zona 1. Las concentraciones de $\mathrm{Pb}, \mathrm{Zn}, \mathrm{Cu}, \mathrm{Fe}$, $\mathrm{Mn}, \mathrm{Ba}$ y As fueron en promedio 2,61, 1,14, 1,40, $2,29,2,31,1,16$ y 1,21 veces mayores en la Zona 2. Los resultados sugirieron que la diferencia en la densidad de tráfico probablemente influyó en la concentración de los elementos metálicos asociados con el sedimento depositado sobre las superficies viarias en evaluación (Zona 1: 2750 vehículos/día; Zona 2: 40100 vehículos/día). Asimismo, Freud y Johnson [22], McKenzie e Irwin [23], y Viklander [24] sugirieron que la deposición de metales pesados sobre las superficies viarias era proporcional a la densidad de tráfico.

Tabla 2. Concentración de metales pesados con un intervalo de confianza del $95 \%$.

\begin{tabular}{|c|c|c|}
\hline \multirow{2}{*}{ Metal } & \multicolumn{2}{|c|}{$\begin{array}{c}\text { Concentración promedio } \\
(\mathrm{mg} / \mathrm{kg} \text { de materia seca) }\end{array}$} \\
\cline { 2 - 3 } & Zona 1 & Zona 2 \\
\hline $\mathrm{Mn}$ & $67,8 \pm 9,6$ & $156,4 \pm 14,4$ \\
\hline $\mathrm{Fe}$ & $8251 \pm 44$ & $18801 \pm 95$ \\
\hline $\mathrm{Ba}$ & $105,1 \pm 12,4$ & $121,4 \pm 11,2$ \\
\hline $\mathrm{Pb}$ & $74,1 \pm 12,5$ & $193,3 \pm 18,3$ \\
\hline $\mathrm{Cd}$ & $0,44 \pm 0,2$ & $0,32 \pm 0,1$ \\
\hline $\mathrm{Cu}$ & $38,7 \pm 8,0$ & $54,0 \pm 11,5$ \\
\hline $\mathrm{Zn}$ & $65,3 \pm 0,7$ & $74,3 \pm 3,7$ \\
\hline $\mathrm{As}$ & \multicolumn{2}{|c|}{$0,92 \pm 0,2$} \\
\hline
\end{tabular}

Nota: ${ }^{a}$ Concentración para la fracción de tamaño menor a $250 \mu \mathrm{m}$. Fuente: elaboración propia.

Se realizó un análisis de correlación entre todos los metales pesados con el objeto de profundizar en la afinidad del origen (i.e., coeficiente de correlación lineal). En la calzada de la Zona 1 existió correlación entre $\mathrm{Pb}, \mathrm{Ba}, \mathrm{Mn}$ y $\mathrm{Cu}$; y entre $\mathrm{Cd}$ y $\mathrm{Fe}$ (tabla 3). Por otro lado, en la Zona 2 existió correlación entre $\mathrm{Pb}, \mathrm{Cu}$, Ba y As; y entre $\mathrm{Zn}, \mathrm{Mn}$ y Fe (tabla 4).

A partir de lo anterior, los resultados sugirieron en las vías de estudio que las principales fuentes para el primer grupo de metales pesados (por ejemplo, $\mathrm{Pb}$, $\mathrm{Ba}, \mathrm{Cu}$ y $\mathrm{Mn}$ ) fueron las partículas desprendidas por el uso de las pastillas de los frenos y de las llantas (caucho). A este primer grupo se le añadirían $\mathrm{Zn}$, $\mathrm{Fe}$, As y Cd por el uso de las pastillas de los frenos. El material de fricción de las pastillas de los frenos 


\section{|investigación}

está constituido por un $15 \%$ de elementos metálicos [25]. En este sentido, Shaheen [26] reportó elevadas concentraciones de $\mathrm{Pb}$ y Cu en materiales como el revestimiento para los frenos $(\mathrm{Pb}: 1050 \mathrm{mg} / \mathrm{kg}$; $\mathrm{Cu}: 30$ $600 \mathrm{mg} / \mathrm{kg}$ ) y el caucho desprendido por el desgaste de las llantas (Pb: 1110 mg/kg; Cu: $247 \mathrm{mg} / \mathrm{kg}$ ).

Tabla 3. Coeficientes de correlación lineal para la concentración entre metales pesados para la fracción de tamaño $<250 \mu \mathrm{m}$. Zona 1 .

\begin{tabular}{|c|c|c|c|c|c|c|c|c|}
\hline Metal & $\mathrm{Mn}$ & $\mathrm{Fe}$ & $\mathrm{Ba}$ & $\mathrm{Pb}$ & $\mathrm{Cd}$ & $\mathrm{Cu}$ & $\mathrm{Zn}$ & $\mathrm{As}$ \\
\hline $\mathrm{Mn}$ & 1,00 & & & & & & & \\
$\mathrm{Fe}$ & 0,34 & 1,00 & & & & & & \\
$\mathrm{Ba}$ & 0,61 & 0,28 & 1,00 & & & & & \\
$\mathrm{~Pb}$ & 0,63 & 0,23 & 0,85 & 1,00 & & & & \\
$\mathrm{Cd}$ & 0,29 & 0,55 & 0,22 & 0,17 & 1,00 & & & \\
$\mathrm{Cu}$ & 0,64 & 0,32 & 0,59 & 0,76 & 0,33 & 1,00 & & \\
$\mathrm{Zn}$ & 0,10 & 0,26 & 0,03 & 0,13 & 0,19 & 0,43 & 1,00 & \\
$\mathrm{As}$ & 0,37 & 0,43 & 0,35 & 0,32 & 0,21 & 0,34 & 0,21 & 1,00 \\
\hline
\end{tabular}

Fuente: elaboración propia.

Tabla 4. Coeficientes de correlación lineal para la concentración entre metales pesados para la fracción de tamaño $<250$ $\mu \mathrm{m}$. Zona 2 .

\begin{tabular}{|c|c|c|c|c|c|c|c|c|}
\hline Metal & $\mathrm{Mn}$ & $\mathrm{Fe}$ & $\mathrm{Ba}$ & $\mathrm{Pb}$ & $\mathrm{Cd}$ & $\mathrm{Cu}$ & $\mathrm{Zn}$ & $\mathrm{As}$ \\
\hline $\mathrm{Mn}$ & 1,00 & & & & & & & \\
$\mathrm{Fe}$ & 0,75 & 1,00 & & & & & & \\
$\mathrm{Ba}$ & $-0,23$ & $-0,42$ & 1,00 & & & & & \\
$\mathrm{~Pb}$ & 0,41 & 0,42 & 0,22 & 1,00 & & & & \\
$\mathrm{Cd}$ & $-0,21$ & $-0,14$ & 0,11 & $-0,22$ & 1,00 & & & \\
$\mathrm{Cu}$ & $-0,09$ & $-0,08$ & 0,60 & 0,64 & 0,01 & 1,00 & & \\
$\mathrm{Zn}$ & 0,55 & 0,53 & $-0,02$ & 0,49 & $-0,28$ & 0,27 & 1,00 & \\
$\mathrm{As}$ & 0,05 & 0,05 & 0,61 & 0,58 & 0,03 & 0,74 & 0,25 & 1,00 \\
\hline
\end{tabular}

Fuente: elaboración propia.

Se realizó una comparación entre las concentraciones metálicas obtenidas y los estándares establecidos por la legislación para los suelos urbanos de los siguientes países: Turquía [27], Italia [28] y Venezuela [29]. De los ocho metales pesados en estudio únicamente se evaluaron el $\mathrm{Pb}, \mathrm{Cd}, \mathrm{Zn}$ y As, puesto que fueron los elementos metálicos que se encontraban regulados por la normatividad de referencia. Los resultados mostraron para la Zona 2 que $\mathrm{Pb}$ superó los límites establecidos, en promedio 1,93, 1,29 y 1,29 veces con respecto de la legislación de Italia $(100 \mathrm{mg} / \mathrm{kg})$, Turquía $(150 \mathrm{mg} / \mathrm{kg})$ y Venezuela $(150 \mathrm{mg} / \mathrm{kg})$, respectivamente (figura 5). Por otro lado, en las Zonas 1 y 2 el Zn superó los límites establecidos por la legislación de Turquía (50 mg/kg) en 1,31 y 1,49 veces, respectivamente (figura 6). Adicionalmente, los resultados mostraron para las Zonas 1 y 2 que las concentraciones de $\mathrm{Pb}$ superaron el límite establecido por la legislación de Italia el $21,7 \%$ y $87,0 \%$ de las veces durante el periodo de muestreo, respectivamente. Por otro lado, los resultados mostraron para las Zonas 1 y 2 que las concentraciones de $\mathrm{Zn}$ superaron el límite establecido por la legislación de Turquía el 84,8 \% y 82,6\% de las veces durante el periodo de estudio, respectivamente.

Por último, los resultados evidenciaron que las concentraciones metálicas asociadas con el sedimento viario tendieron a disminuir durante la época de aumento de la precipitación (i.e., probablemente por lavado de la escorrentía). En este sentido, posiblemente existió relación entre el volumen de precipitación y la concentración metálica asociada con el sedimento viario (figuras 4 y 5). Sin embargo, en promedio para todos los metales pesados en estudio, el coeficiente de correlación lineal dio evidencia de una relación negativa débil $(\mathrm{r}=-0,24)$.

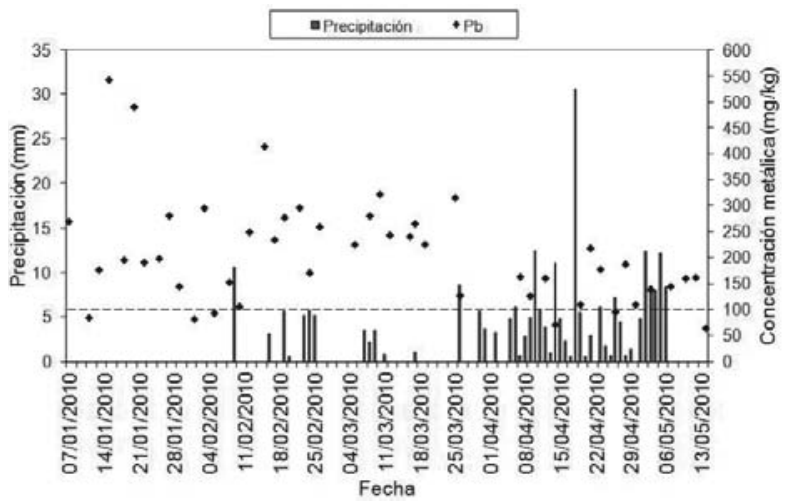

Figura 5. Comparación entre la concentración de $\mathrm{Pb}$ en el sedimento viario y la normatividad internacional de referencia para el suelo urbano (Italia). Zona 2.

Fuente: elaboración propia. 


\section{investigación |}

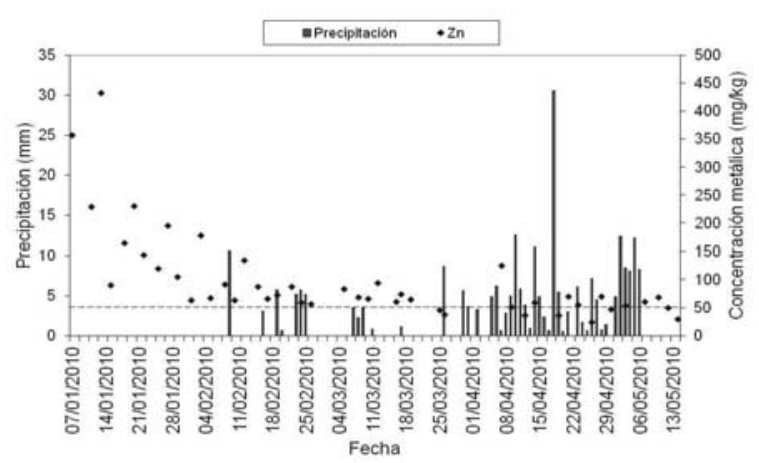

Figura 6. Comparación entre la concentración de Zn en el sedimento viario y la normatividad internacional de referencia para el suelo urbano (Turquía). Zona 2.

Fuente: elaboración propia.

\section{CONCLUSIONES}

Los resultados mostraron que las concentraciones de $\mathrm{Pb}$ y $\mathrm{Zn}$ asociadas con el sedimento viario están superando los límites establecidos por la legislación utilizada como de referencia para suelos urbanos: Italia y Turquía, respectivamente. De esta manera, el $\mathrm{Pb}$ y $\mathrm{Zn}$ se destacan como los elementos metálicos de mayor atención para las autoridades encargadas del control ambiental sobre las áreas de estudio (por ejemplo, de las ciudades de Soacha y Bogotá), puesto que el sedimento viario podría ser catalogado como un residuo peligroso.
Por otro lado, los resultados sugieren en las zonas de estudio que el origen de los metales pesados asociados con el sedimento viario son las fuentes móviles, principalmente por partículas desprendidas por el uso de las pastillas de los frenos y por el desgaste de las llantas (caucho).

Finalmente, las anteriores consideraciones podrán ser de utilidad para las instituciones encargadas de la gestión de la contaminación superficial, para diseñar y evaluar las prácticas de control de la contaminación metálica presente sobre las superficies viarias de investigación, como por ejemplo el establecimiento de la frecuencia del barrido viario en la época seca y de lluvias. Adicionalmente, la investigación se constituye en un punto de referencia a nivel nacional para el desarrollo y promulgación de legislación ambiental para el control de este tipo de contaminantes en áreas urbanas.

\section{FINANCIAMIENTO}

Los autores desean agradecer el apoyo financiero brindado por el Laboratorio Ambiental y la Dirección de Calidad del Aire de la Corporación Autónoma Regional de Cundinamarca (CAR) (Colombia), y del Centro de Investigación y Desarrollo Científico de la Universidad Distrital Francisco José de Caldas (Colombia).

\section{REFERENCIAS}

[1] M. H. Hermanson, "Anthropogenic Mercury Deposition to Arctic Lake Sediments", Water, Air and Soil Pollution, vol. 101, no. 1-4, pp. 309-321, Jan. 1998.

[2] A. Fernández, M. Ternero, F. Barragán and J. Jiménez, "An approach to characterization of sources of urban airborne particles through heavy metal speciation", Chemosphere, vol. 2, no. 2, pp. 123-136, Apr. 2000.
[3] K. Johansson, M. Aastrup, A. Andersson, L. Bringmark and A. Iverfeldt, "Mercury in swedish forest soils and waters-Assessment of critical load", Water, Air and Soil Pollution, vol. 56, no. 1, pp. 267-281, Jan. 1991.

[4] J. Ford et ál., "Inorganic contaminants in Arctic Alaskan ecosystems: long-range atmospheric transport or local point sources", 
Science of The Total Environment, vol. 160, no. 1, pp. 323-335, Jan. 1995.

[5] The World Bank, Environmental Priorities and Poverty Reduction: a Country Environmental Analysis for Colombia. Washington D.C.: The World Bank, 2007.

[6] C. Saskia, V. Zee, H. Harssema and B. Brunekreef, "Characterization of particulate air pollution in urban and non-urban in the Netherlands", Atmospheric Environment, vol. 32, no. 21, pp. 3717-3729, Nov. 1998.

[7] J.E. Ball, R. Jenks and D. Aubourg, "An assessment of the availability of pollutant constituents on road surfaces", Science of The Total Environment, vol. 209, no. 2-3, pp. 243-254, Feb.1998.

[8] M. Berhanu, M. Bruen, N. Higgins and P. Johnston, "Highway runoff quality in Ireland", Journal of Environmental Monitoring, vol. 9, no. 4, pp. 366-371, Apr. 2007.

[9] B. Wei and L. Yang, "A review of heavy metal contaminations in urban soils, urban road dusts and agricultural soils from China", Microchemical Journal, vol. 94, no. 2 , pp. 99-107, Feb. 2010.

[10] S. Constantini and V. Demetra, "Size distribution of airborne particulate matter and associated heavy metals in the roadside environment", Chemosphere, vol. 59, no. 8, pp. 1197-1206, Aug. 2005.

[11] C.A. Zafra, J. Temprano and I. Tejero, "Distribution of the concentration of heavy metals associated with the sediment particles accumulated on road surfaces", Environmental Technology, vol. 32, no. 9, pp. 997-1008, Jul. 2011.

[12] V. Novotny and H. Olem, Water quality: prevention, identification and management

\section{investigación}

of diffuse pollution. New York: Van Nostrand Reinhold, 1994.

[13] P. Malgrat, Panorámica general de la escorrentía de aguas pluviales como fuente de contaminación, Actuaciones posibles, Jornadas Técnicas sobre la Calidad de Aguas e Impacto en Medios Receptores por Vertidos Procedentes de Drenajes Urbanos y Agrícolas. Castellón: University Jaume I, 1995.

[14] C.A. Zafra, J. Temprano and I. Tejero, "Particle size distribution of accumulated sediments on an urban road in rainy weather", Environmental Technology, vol. 29, no. 5, pp. 571-582, May. 2008.

[15] C.A. Zafra, J. Temprano y I. Tejero, "Contaminación por escorrentía superficial urbana: metales pesados acumulados sobre la superficie de una vía", Ingeniería e Investigación, vol. 27, no. 1, pp. 4-10, abril 2007.

[16] D.K. Essumang, D.K. Dodoo, S. Obiri and B.A.K. Oduro, "Analysis of vehicular fallouts from traffic in the Kumasi Metropolis, Ghana", Bulletin of the Chemical Society of Ethiopia, vol. 20, no. 1, pp. 9-15, Jun. 2006.

[17] American Society for Testing and Materials, Standard test method for laboratory determination of water (moisture) content of soil and rock by mass, ASTM D2216. West Conshohocken: ASTM, 2000.

[18] International Organization for Standardization, ISO Soil quality, Extraction of trace elements soluble in aqua regia, ISO 11466. Geneva: ISO, 2000.

[19] International Organization for Standardization, ISO Soil quality, Determination of cadmium, chromium, cobalt, copper, lead, manganese, nickel and zinc, Flame and 


\section{investigación |}

electrothermal atomic absorption spectrometric methods, ISO 11047. Geneva: ISO, 2000 .

[20] M.P. Barkdoll, D.E. Overton and R.P. Betson, "Some effects of dustfall on urban stormwater quality", Water Pollution Control Federation, vol. 49, no. 9, pp. 19761984, Sep. 1977.

[21] A. Machado et ál., "Contaminación por metales $(\mathrm{Pb}, \mathrm{Zn}$, Ni y $\mathrm{Cr}$ ) en aire, sedimentos viales y suelo en una zona de alto tráfico vehicular", Revista Internacional de Contaminación Ambiental, vol. 24, no. 4, pp. 171-182, abril 2008.

[22] A.P. Freud and C.D. Johnson, "Comparison and relationships of stormwater quality and basin characteristics: Madison, Wisconsin", Presented at the Proceeding of the International Symposium on Urban Storm Runoff, Kentucky, USA, Jul. 1980.

[23] D.J. McKenzie and G.A. Irwin, Water-quality assessment of stormwater runoff from a heavily used urban highway bridge in Miami, Florida, Menlo Park, California, Tech. Rep. 83-4153, 1983.
[24] M. Viklander, "Particle size distribution and metal content in street sediments", Journal of Environmental Engineering, vol. 124, no. 8, pp. 761-766, Aug. 1998.

[25] RoadHouse, Brake pads technical manual, Arganda del Rey: RoadHouse, 2008.

[26] D.G. Shaheen, Contributions of urban roadway usage to water pollution, Washington D.C., Tech. Rep. EPA600/2-75-004, 1975.

[27] Turkish Goverment, The Solid Waste Pollution Control Regulation (TSPCR), Istanbul: Ministry of Environment, 2000.

[28] Gazzetta Ufficiale della Repubblica Italiana, Regolamento recante criteri, procedure e modalita per la messa in sicurezza, la bonifica e il ripristino ambientale dei siti inquinati, Rome: Gazzetta Ufficiale della Repubblica Italiana, 1999.

[29] Gaceta Oficial Venezolana, Normas para el Control de la Recuperación de Materiales Peligrosos y el Manejo de los desechos Peligrosos, Caracas: Gaceta Oficial Venezolana, 1998. 